Pacific

Journal of

Mathematics

FREE FISHER INFORMATION FOR NON-TRACIAL STATES

Dimitri SHLYAKHTENKO 


\title{
FREE FISHER INFORMATION FOR NON-TRACIAL STATES
}

\author{
DimitRi SHLYAKHTENKO
}

\begin{abstract}
We extend Voiculescu's microstates-free definitions of free Fisher information and free entropy to the non-tracial framework. We explain the connection between these quantities and free entropy with respect to certain completely positive maps acting on the core of the non-tracial non-commutative probability space. We give a condition on free Fisher information of an infinite family of variables, which guarantees factoriality of the von Neumann algebra they generate.
\end{abstract}

\section{Introduction.}

Free entropy and free Fisher information were introduced by Voiculescu [13], $[\mathbf{1 4}]$ and $[\mathbf{1 6}]$ in the context of his free probability theory [17] as analogs of the corresponding classical quantities. These quantities are usually considered in the framework of tracial non-commutative probability spaces; not surprisingly, the most striking applications of free entropy theory were to tracial von Neumann algebras (see e.g., [15], [2] and [10]). Recently, however, it turned out that some type III factors associated with free probability theory [5] have certain properties in common with their type $\mathrm{II}_{1}$ cousins [9]. This gives rise to a speculation that there is room for free entropy to exist outside of the context of tracial non-commutative probability spaces.

The goal of this paper is to initiate the development of free Fisher information, based on Voiculescu's microstates-free approach [16], in the non-tracial framework. The key idea is that all of the ingredients going into the definition of free Fisher information in this case must behave covariantly with respect to the modular group [11] of the non-tracial state. The principal example of a family of variables for which free Fisher information is nontrivial, and which belong to an algebra not having any traces, are semicircular generators of free Araki-Woods factors, taken with free quasi-free states [5].

We describe another route towards free Fisher information, which is based on first converting the non-tracial von Neumann algebra into a larger algebra, the core (having an infinite trace), and then considering free Fisher information relative to a certain completely positive map (in the spirit of [7]). We should point out that it is this approach that is most likely to connect with the microstates free entropy (as suggested by [3]; see also [8]), 
since it is at present unclear what a microstates approach to free entropy in the non-tracial framework should be.

We finish the paper with a look at free Fisher information on von Neumann algebras that have traces. Our first result is that once the algebra has a trace, the free Fisher information is automatically infinite when computed with respect to a non-tracial state. It is likely that on any von Neumann algebra, free Fisher information can be finite for only very special states (however, we do not have any results in this direction in the non-tracial category). Another result of the present paper is a statement guaranteeing factoriality of a tracial von Neumann algebra, once we know that it has an infinite generating family whose free Fisher information is bounded in a certain way.

\section{Free Fisher information for arbitrary KMS states.}

2.1. Free Brownian motion in the presence of a modular group. Let $M$ be a von Neumann algebra, $\phi: M \rightarrow \mathbb{C}$ be a normal faithful state on $M$. Denote by $\sigma_{t}^{\phi}$ the modular group of $\phi$. Denote by $L^{2}\left(M^{\mathrm{sa}}, \phi\right) \subset L^{2}(M, \phi)$ the closure of the real subspace of self-adjoint elements $M^{\text {sa }} \subset M$.

Let $X=X^{*} \in M$ and let $B \subset M$ be a subalgebra. Assume that $\sigma_{t}^{\phi}(B) \subset$ $B$ for all $t \in \mathbb{R}$.

Consider the von Neumann algebra $\mathcal{M}=\Gamma\left(L^{2}\left(M^{\text {sa }}, \phi\right) \subset L^{2}(M, \phi)\right)$, taken with the free quasi-free state $\phi_{\mathcal{M}}$. Consider the element $Y=s(X) \in$ $\mathcal{M}$ (see [5] for definitions and notation). Then $\phi_{\mathcal{M}}\left(Y \sigma_{t}^{\phi_{\mathcal{M}}}(Y)\right)=\phi\left(X \sigma_{t}^{\phi}(X)\right)$, for all $t \in \mathbb{R}$.

Consider the algebra $\mathcal{N}=(M, \phi) *\left(\mathcal{M}, \phi_{\mathcal{M}}\right)$, and denote by $\hat{\phi}$ the free product state on $\mathcal{N}$. Note that $\sigma_{t}^{\hat{\phi}}=\sigma_{t}^{\phi} * \sigma_{t}^{\phi} \mathcal{M}$. The elements $X_{\varepsilon}=X+\sqrt{\varepsilon} Y$, $\varepsilon \geq 0$ form a natural free Brownian motion, which behaves nicely under the action of the modular group. In particular, note that for all $\varepsilon \geq 0$ and $t \in \mathbb{R}$,

$$
\hat{\phi}\left(X_{\varepsilon} \sigma_{t}^{\hat{\phi}}\left(X_{\varepsilon}\right)\right)=\phi\left(X \sigma_{t}^{\phi}(X)\right) \cdot(1+\varepsilon) .
$$

Furthermore, for each $\varepsilon>0$, the distribution of $X_{\varepsilon}$ is that of a free Brownian motion at time $\varepsilon$, starting at $X$; this is because $Y$ is a semicircular variable, free from $X$.

2.2. Conjugate variables. Let $B[X]$ denote that algebra generated by $B$ and all translates $\sigma_{t}^{\phi}(X), t \in \mathbb{R}$. Assume that $\left\{\sigma_{t}^{\phi}(X)\right\}$ are algebraically free over $B$, i.e., satisfy no algebraic relations modulo $B$. Denote by $\partial_{X}$ : $B[X] \rightarrow \mathcal{N}$ the derivation given by:

1. $\partial_{X}\left(\sigma_{t}^{\phi}(X)\right)=\sigma_{t}^{\hat{\phi}}(Y)$

2. $\partial_{X}(b)=0, b \in B$.

Notice that the range of $\partial_{X}$ actually lies in the subspace $B[X] \cdot \operatorname{span}\left\{\sigma_{t}^{\hat{\phi}}(Y)\right.$ : $t \in \mathbb{R}\} \cdot B[X] \subset \mathcal{N}$. Note also that since $\partial_{X}\left(\sigma_{t}^{\phi}(X)\right)$ is self-adjoint, we have 
that for $P \in B[X], \partial_{X}\left(P^{*}\right)=\partial_{X}(P)^{*}$, i.e., $\partial_{X}$ is a $*$-derivation. Observe finally that $\partial_{X}$ is covariant with respect to the modular groups $\sigma_{t}^{\phi}$ and $\sigma_{t}^{\hat{\phi}}$ :

$$
\partial_{X}\left(\sigma_{t}^{\phi}(P)\right)=\sigma_{t}^{\hat{\phi}}\left(\partial_{X}(P)\right), \quad P \in B[X] .
$$

Define the conjugate variable $J_{\phi}(X: B) \in L^{2}(B[X], \phi)$ to be such a vector $\xi$ that

$$
\langle\xi, P\rangle_{L^{2}(B[X], \phi)}=\left\langle Y, \partial_{X}(P)\right\rangle_{L^{2}(\mathcal{N}, \hat{\phi})}, \quad \forall P \in B[X],
$$

if a vector $\xi$ satisfying such properties exists. Formally, this means that $\xi=\partial_{X}^{*}(Y)$, where $\partial_{X}: L^{2}(B[X], \phi) \rightarrow L^{2}(\mathcal{N}, \hat{\phi})$ is viewed as a densely defined operator.

It is clear, because of the density of $B[X]$ in $L^{2}(B[X], \phi)$, that $\xi$ is unique, if it exists.

It is convenient to talk about $J_{\phi}(X: B)$ even in the case that $\left\{\sigma_{t}(X)\right\}_{t \in \mathbb{R}}$ are not algebraically free over $B$ (such is the case, for example, when $\phi$ is a trace, and hence $\sigma_{t}^{\phi}(X)=X$ for all $t$ ). In this case, one can view $\partial_{X}$ as a multi-valued map, the set of values given by the results of application of the definition of $\partial_{X}$ in all possible ways; the definition of $J_{\phi}$ is then that (2.1) is valid for all values of $\partial_{X}$.

Note that $J_{\phi}(X: B)$ depends on more than just the joint distribution of $X$ and $B$ with respect to the state $\phi$; it depends on the joint distribution of the family $B \cup\left\{\sigma_{t}^{\phi}(X): t \in \mathbb{R}\right\}$.

We continue to denote by $\sigma_{t}^{\phi}$ the extension of $\sigma_{t}^{\phi}$ to the Hilbert space $L^{2}(M, \phi)$ (this is precisely the one-parameter group of unitaries $\Delta_{\phi}^{i t}$, where $\Delta_{\phi}$ is the modular operator). In particular, if $\phi$ is a trace, then the definition of $J_{\phi}$ is (up to a multiple) precisely that of the conjugate variable of Voiculescu [16].

Lemma 2.1. Assume that $\xi=J_{\phi}(X: B)$ exists. Then $\xi \in L^{2}\left(M^{\mathrm{sa}}, \phi\right)$, and $\sigma_{t}^{\phi}\left(J_{\phi}(X: B)\right)=J_{\phi}\left(\sigma_{t}^{\phi}(X): B\right)$.

Proof. We note that, because $\partial_{X}$ is a $*$-derivation,

$$
\left\langle P^{*}, \xi\right\rangle=\overline{\left\langle Y, \partial_{X}\left(P^{*}\right)\right\rangle}=\left\langle\partial_{X}(P), Y\right\rangle=\overline{\langle\xi, P\rangle} .
$$

From this it follows that $\xi$ is in the domain of the $S$ operator of Tomita theory, and moreover that $S \xi=\xi$. Hence $\xi \in L^{2}\left(M^{\text {sa }}\right)$.

One also has

$$
\begin{aligned}
\left\langle\sigma_{t}^{\phi}(\xi), P\right\rangle & =\left\langle Y, \partial_{X}(P)\right\rangle \\
& =\left\langle\sigma_{t}^{\phi}(Y), \partial_{\sigma_{t}^{\phi}(X)}(P)\right\rangle,
\end{aligned}
$$

since the joint distributions of $B[X]$ and $\left\{\sigma_{t}^{\hat{\phi}}(Y)\right\}_{t \in \mathbb{R}}$ is the same as $B[X]$ and $\left\{\sigma_{s+t}^{\hat{\phi}}(Y)\right\}_{t \in \mathbb{R}}$, for any $s$. It follows that $\sigma_{t}^{\phi}\left(J_{\phi}(X: B)\right)=J_{\phi}\left(\sigma_{t}^{\phi}(X): B\right)$. 
Lemma 2.2. Let $P, Q \in B[X]$, and assume that $\xi=J_{\phi}(X)$ exists and is in M. Then

$$
\phi(P \xi Q)=\hat{\phi}\left(P Y \partial_{X}(Q)\right)+\hat{\phi}\left(\partial_{X}(P) Y Q\right) .
$$

Proof. Recall that $\phi$ (and $\hat{\phi}$ ) satisfy the KMS condition: For all $a, b \in M$ (or $\in \mathcal{N}$ ), there exists a (unique) function $f(z)$, analytic on the strip $\{z$ : $0<\Im z<1\}$, and so that (writing $\sigma_{t}$ for either $\sigma_{t}^{\phi}$ or $\sigma_{t}^{\hat{\phi}}$ )

$$
\begin{aligned}
& \phi\left(a \sigma_{t}(b)\right)=f(t), \\
& \phi\left(\sigma_{t}(b) a\right)=f(t+i), \quad t \in \mathbb{R} .
\end{aligned}
$$

Fix $P, Q \in B[X]$ and let $f$ be as above, so that

$$
\begin{aligned}
\phi\left(\sigma_{t}^{\phi}(P) \xi Q\right) & =f(t+i), \\
\phi\left(\xi Q \sigma_{t}^{\phi}(P)\right) & =f(t) .
\end{aligned}
$$

Then

$$
\begin{aligned}
f(t) & =\left\langle\xi, Q \sigma_{t}^{\phi}(P)\right\rangle \\
& =\left\langle Y, \partial_{X}\left(Q \sigma_{t}^{\phi}(P)\right)\right\rangle \\
& =\left\langle Y, \partial_{X}(Q) \sigma_{t}^{\phi}(P)\right\rangle+\left\langle Y, Q \sigma_{t}^{\hat{\phi}}\left(\partial_{X}(P)\right)\right\rangle,
\end{aligned}
$$

where in the last step we used the fact that $\partial_{X}$ intertwines $\sigma_{t}^{\phi}$ and $\sigma_{t}^{\hat{\phi}}$. Using the KMS-condition for $\hat{\phi}$, we then get

$$
\begin{aligned}
f(t+i) & =\hat{\phi}\left(\sigma_{t}^{\hat{\phi}}(P) Y \partial_{X}(Q)\right)+\hat{\phi}\left(\sigma_{t}^{\hat{\phi}}\left(\partial_{X}(P)\right) Y Q\right) \\
& =\hat{\phi}\left(\sigma_{t}^{\hat{\phi}}(P) Y \partial_{X}(Q)\right)+\hat{\phi}\left(\partial_{X}\left(\sigma_{t}^{\hat{\phi}}(P)\right) Y Q\right) .
\end{aligned}
$$

Since $f(t+i)=\phi\left(\sigma_{t}^{\hat{\phi}}(P) \xi Q\right)$, we get, setting $t=0$, that

$$
\phi(P \xi Q)=\hat{\phi}\left(P Y \partial_{X}(Q)\right)+\hat{\phi}\left(\partial_{X}(P) Y Q\right),
$$

as claimed.

2.3. Conjugate variables as free Brownian gradients. As pointed out above, $X+\sqrt{\varepsilon} Y$ is a natural free Brownian motion, which is covariant with respect to the appropriate modular groups. The following proposition shows that $J_{\phi}(X: B)$ plays the role of the free Brownian gradient of $X$ :

Proposition 2.3. Assume that $\xi=J_{\phi}(X: B)$ exists and belongs to $M \subset$ $L^{2}(M, \phi)$. Let $P\left(Z_{1}, \ldots, Z_{n}\right)$ be any non-commutative polynomial in $n$ variables $Z_{1}, \ldots, Z_{n}$, with coefficients from $B$. Write $X_{t}=\sigma_{t}^{\phi}(X), Y_{t}=\sigma_{t}^{\hat{\phi}}(Y)$, $\xi_{t}=\sigma_{t}^{\phi}(\xi), t \in \mathbb{R}$.

Then for all $t_{1}, \ldots, t_{n} \in \mathbb{R}$, we have

$$
\begin{aligned}
& \hat{\phi}\left(P\left(X_{t_{1}}+\sqrt{\varepsilon} Y_{t_{1}}, \ldots, X_{t_{n}}+\sqrt{\varepsilon} Y_{t_{n}}\right)\right) \\
& =\frac{1}{2} \phi\left(P\left(X_{t_{1}}+\varepsilon \xi_{t_{1}}, \ldots, X_{t_{n}}+\varepsilon \xi_{t_{n}}\right)\right)+O\left(\varepsilon^{2}\right) .
\end{aligned}
$$


Proof. We may assume, by linearity, that $P$ is a monomial, i.e., $P\left(Z_{1}, \ldots\right.$, $\left.Z_{n}\right)=b_{0} Z_{1} b_{1} \ldots b_{n-1} Z_{n} b_{n}$, for $b_{j} \in B$. In this case, we have

$$
\begin{aligned}
\hat{\phi} & \left(b_{0}\left(X_{t_{1}}+\sqrt{\varepsilon} Y_{t_{1}}\right) b_{1} \ldots b_{n}\right) \\
= & \phi\left(P\left(X_{t_{1}}, \ldots, X_{t_{n}}\right)\right)+O\left(\varepsilon^{2}\right) \\
& +\varepsilon \sum_{k<l} \hat{\phi}\left(b_{0} X_{t_{1}} \ldots b_{l} Y_{t_{l+1}} b_{l+1} X_{t_{l+2}} \ldots b_{k} Y_{t_{k+1}} b_{k+1} X_{t_{k+2}} \ldots X_{t_{n}} b_{n}\right) \\
= & \phi\left(P\left(X_{t_{1}}, \ldots, X_{t_{n}}\right)\right)+O\left(\varepsilon^{2}\right) \\
& +\frac{1}{2} \varepsilon \sum_{l} \hat{\phi}\left(b_{0} X_{t_{1}} \ldots X_{t_{l}} b_{l} Y_{t_{l+1}} \partial_{X_{t_{l+1}}}\left(b_{l+1} X_{t_{l+2}} \ldots X_{t_{n}} b_{n}\right)\right) \\
& +\frac{1}{2} \varepsilon \sum_{k} \hat{\phi}\left(\partial_{X_{t_{k+1}}}\left(b_{0} X_{t_{1}} \ldots X_{t_{k}} b_{k}\right) Y_{t_{k}} b_{k+1} X_{t_{k+2}} \ldots X_{t_{n}} b_{n}\right) \\
= & \phi\left(P\left(X_{t_{1}}, \ldots, X_{t_{n}}\right)\right)+O\left(\varepsilon^{2}\right) \\
& +\frac{1}{2} \sum_{k} \phi\left(b_{0} X_{t_{1}} \ldots X_{t_{k}} b_{k} \varepsilon \xi_{t_{k}} b_{k+1} X_{t_{k+2}} \ldots X_{t_{n}} b_{n}\right)
\end{aligned}
$$

the last equality by Lemma 2.2 .

\subsection{Examples of conjugate variables.}

2.4.1. Tracial case. We have seen before that if $\phi$ is a trace, then the definition of $J_{\phi}(X: B)$ coincides with the definition of conjugate variables given by Voiculescu, up to a constant (which has to do with the fact that we choose $Y$ so that $\|Y\|_{L^{2}(\hat{\phi})}=\|X\|_{L^{2}(\phi)}$, and not 1). In particular,

$$
J_{\phi}(X: B)=J(X: B) \cdot \frac{1}{\|X\|_{L^{2}(\phi)}^{2}}, \quad \text { if } \phi \text { is a trace. }
$$

2.4.2. Free quasi-free states. Let $\mu$ be a positive finite Borel measure on $\mathbb{R}$, so that $\mu$ is symmetric, $\mu(-X)=\mu(X)$ for all Borel subsets $X \subset \mathbb{R}$. Let $\mathcal{H}_{\mathbb{R}}$ be the real Hilbert space of $\mu$-square-integrable functions, satisfying $f(-x)=\overline{f(x)}$ for all $x \in \mathbb{R}$. Denote by $U_{t}$ the representation of $\mathbb{R}$ on $\mathcal{H}_{\mathbb{R}}$, given by

$$
\left(U_{t} f\right)(x)=e^{2 \pi i t x} f(x), \quad x, t \in \mathbb{R} .
$$

Let $h$ denote the vector $1 \in \mathcal{H}_{\mathbb{R}}$, and consider

$$
M=\Gamma\left(\mathcal{H}_{\mathbb{R}}, U_{t}\right)^{\prime \prime}, \quad \phi=\phi_{U}, \quad X=s(h) \in M
$$

(see [5] for definitions and notation). 
Then $X=J_{\phi}(X: \mathbb{C})$. Indeed, set $X_{t}=\sigma_{t}^{\phi}(X)=s\left(U_{t} h\right)$; then we have

$$
\begin{aligned}
\phi\left(X \cdot X_{t_{1}} \ldots X_{t_{n}}\right) & =\sum_{k} \phi\left(X X_{t_{k}}\right) \phi\left(X_{t_{1}} \ldots X_{t_{k-1}}\right) \cdot \phi\left(X_{t_{k+1}} \ldots X_{t_{n}}\right) \\
& =\sum_{k} \hat{\phi}\left(Y \sigma_{t_{k}}^{\hat{\phi}}(Y)\right) \phi\left(X_{t_{1}} \ldots X_{t_{k-1}}\right) \cdot \phi\left(X_{t_{k+1}} \ldots X_{t_{n}}\right) \\
& =\sum_{k} \hat{\phi}\left(Y X_{t_{1}} \ldots X_{t_{k-1}} Y X_{t_{k+1}} \ldots X_{t_{n}}\right) \\
& =\hat{\phi}\left(Y \partial_{X}\left(X_{t_{1}} \ldots X_{t_{n}}\right)\right)
\end{aligned}
$$

so that $X$ satisfies the defining property of $J_{\phi}(X: \mathbb{C})$, and hence $J_{\phi}(X: \mathbb{C})$ exists and equals $X$.

2.5. Free Fisher information. Following [16], we define the free Fisher information $\Phi_{\phi}^{*}(X: B)$ to be

$$
\Phi_{\phi}^{*}(X: B)=\left\|J_{\phi}(X: B)\right\|_{2}^{2} \cdot\|X\|_{2}^{-2}
$$

(the extra factor $\|X\|_{2}^{-2}$ comes from the fact that $\partial_{X}(X)$ does not have unit norm in our definition). For several variables, we set

$$
\begin{aligned}
& \Phi_{\phi}^{*}\left(X_{1}, \ldots, X_{n}\right) \\
& =\sum \Phi_{\phi}^{*}\left(X_{i}: W^{*}\left(\sigma_{t_{1}}^{\phi}\left(X_{1}\right), \ldots, \hat{X}_{i}, \ldots, \sigma_{t_{n}}^{\phi}\left(X_{n}\right): t_{1}, \ldots, t_{n} \in \mathbb{R}\right)\right)
\end{aligned}
$$

(here $\hat{X}_{i}$ means that $X_{i}$ is omitted).

\section{Free Fisher information relative to the core.}

Recall [11] that if $(M, \phi)$ is as above, its core is defined to be the von Neumann algebra crossed product $P=M \rtimes_{\sigma^{\phi}} \mathbb{R}$. There is a canonical inclusion $M \subset P$, and $P$ is densely spanned by elements of the form

$$
m U_{t}, \quad t \in \mathbb{R},
$$

where $m \in M$, and $U_{t}$ satisfy $U_{t} m U_{t}^{*}=\sigma_{t}^{\phi}(m)$. The elements $U_{t}: t \in \mathbb{R}$ generate a copy of the group von Neumann algebra $L(\mathbb{R}) \subset P$; the map

$$
E^{\phi}: m U_{t} \mapsto \phi(m) U_{t}, \quad m \in M, t \in \mathbb{R}
$$

extends to a normal conditional expectation from $P$ onto $L(\mathbb{R})$.

For $X \in M$ self-adjoint, define the completely positive map $\eta_{X}: L(\mathbb{R}) \rightarrow$ $L(\mathbb{R})$ by

$$
\eta_{X}(g)=E^{\phi}(X g X), \quad g \in L(\mathbb{R}) .
$$

Identify $L(\mathbb{R})$ with $L^{\infty}(\mathbb{R})$ via Fourier transform. For each $t \in \mathbb{R}$, set

$$
\eta(t)=\left\langle X, \sigma_{t}^{\phi}(X)\right\rangle=E^{\phi}\left(X U_{t} X\right) .
$$

Then $\eta_{X}(f)=\hat{\eta} * f$, if $f \in L^{\infty}(\mathbb{R}) \cong L(\mathbb{R})$; here $\hat{\eta}$ denotes Fourier transform. 
Define on $P$ an $L(\mathbb{R})$-valued inner product

$$
\langle a, b\rangle_{L(\mathbb{R})}=E^{\phi}\left(a^{*} b\right), \quad a, b \in P .
$$

Denote by $L^{2}\left(P, E^{\phi}\right)$ the $L(\mathbb{R})$-Hilbert bimodule arising from the completion of $P$ with respect to the norm induced by this inner product. Note that the restriction of $\langle\cdot, \cdot\rangle_{L(\mathbb{R})}$ to $M \subset P$ is valued in the complex field, and coincides with the inner product $\langle a, b\rangle=\phi\left(a^{*} b\right)$ on $L^{2}(M)$.

Denote by $\langle\cdot, \cdot\rangle_{\eta}$ the $L(\mathbb{R})$-valued inner product on $P \otimes P$ (algebraic tensor product) given by

$$
\left\langle a \otimes b, a^{\prime} \otimes b^{\prime}\right\rangle_{\eta}=E^{\phi}\left(b^{*} \eta\left(E^{\phi}\left(a^{*} a^{\prime}\right) b^{\prime}\right)\right), \quad a, a^{\prime}, b, b^{\prime} \in P .
$$

Denote by $1 \otimes_{\eta} 1$ the vector $1 \otimes 1 \in P \otimes P$.

Let $\delta_{X}: B[X] \cdot L(\mathbb{R}) \rightarrow P \otimes P$ be determined by

$$
\delta_{X}(X)=1 \otimes_{\eta} 1, \quad \delta_{X}(B \cdot L(\mathbb{R}))=0
$$

and the fact that $\delta_{X}$ is a derivation.

Theorem 3.1. Let $(M, \phi)$ be as above, and let $P$ be its core. Let $i$ : $L^{2}(M, \phi) \rightarrow L^{2}\left(P, E^{\phi}\right)$ be the extension of the inclusion of $M \subset P$. Then $\zeta=i\left(J_{\phi}(X: B)\right)$ satisfies

$$
\langle\zeta, Q\rangle_{L(\mathbb{R})}=\left\langle 1 \otimes_{\eta} 1, \delta_{X}(Q)\right\rangle_{\eta_{X}}
$$

for all $Q \in B[X] \vee L(\mathbb{R})$. Conversely, if there exists a vector $\zeta \in L^{2}\left(P, E^{\phi}\right)$, so that (3.1) is satisfied, then $J_{\phi}(X: B)$ exists and $\zeta=i\left(J_{\phi}(X: B)\right)$.

Proof. Assume first that $J_{\phi}(X: B)$ exists. Set $\zeta=i\left(J_{\phi}(X: B)\right)$. We must verify that (3.1) holds. By linearity, and the fact that $L(\mathbb{R}) B L(\mathbb{R}) \subset B L(\mathbb{R})$, it is sufficient to consider the case when $Q=b_{0} U^{s_{1}} X_{t_{1}} b_{1} U^{s_{2}} \ldots X_{t_{n}} b_{n} U^{s_{n}}$, with $b_{j} \in B$ and $X_{t}=\sigma_{t}^{\phi}(X)$. Then $Q=P \cdot U^{r}$, where $r=\sum s_{j}$, and $P=b_{0} X_{t_{1}^{\prime}} b_{1}^{\prime} \ldots b_{n}^{\prime}$, with $b_{j}^{\prime}=\sigma_{s_{j-1}}^{\phi} \circ \ldots \sigma_{s_{1}}^{\phi}\left(b_{j}\right), t_{j}^{\prime}=s_{j-1}+\cdots+s_{1}+t_{j}$. Note that for $x, y, x^{\prime}, y^{\prime} \in P,\left\langle x \otimes y, x^{\prime} \otimes U y^{\prime}\right\rangle_{\eta}=\left\langle x \otimes y, x^{\prime} U \otimes y\right\rangle_{\eta}$, and $\left\langle x \otimes y, U^{r}\left(x^{\prime} \otimes y^{\prime}\right) U^{s}\right\rangle_{\eta}=\left\langle U^{-r} x \otimes y U^{-s}, x^{\prime} \otimes y^{\prime}\right\rangle_{\eta}$. Using this, we get

$$
\begin{aligned}
& \langle\zeta, Q\rangle_{L(\mathbb{R})} \\
& =\langle\zeta, P\rangle_{L(\mathbb{R})} g \\
& =\langle\zeta, P\rangle_{L^{2}(M, \phi)} \cdot U^{r} \\
& =\hat{\phi}\left(Y \partial_{X}(P)\right) \cdot U^{r} \\
& =\sum_{j} \phi\left(b_{0}^{\prime} X_{t_{1}^{\prime}} \ldots X_{t_{j}^{\prime}} b_{j}^{\prime}\right) \phi\left(b_{j+1}^{\prime} X_{t_{j+2}^{\prime}} \ldots X_{t_{n}^{\prime}} b_{n}^{\prime}\right) \cdot \hat{\phi}\left(Y Y_{t_{j}}\right) U^{r} \\
& =\sum_{j} \phi\left(b_{0}^{\prime} X_{t_{1}^{\prime}} \ldots X_{t_{j}^{\prime}} b_{j}^{\prime}\right) \phi\left(b_{j+1}^{\prime} X_{t_{j+2}^{\prime}} \ldots X_{t_{n}^{\prime}} b_{n}^{\prime}\right) \cdot \phi\left(X U^{t_{j}^{\prime}} X U^{-t_{j}^{\prime}}\right) U^{r} \\
& =\sum_{j} \phi\left(b_{0}^{\prime} X_{t_{1}^{\prime}} \ldots X_{t_{j}^{\prime}} b_{j}^{\prime}\right) \phi\left(b_{j+1}^{\prime} X_{t_{j+2}^{\prime}} \ldots X_{t_{n}^{\prime}} b_{n}^{\prime}\right) \cdot E^{\phi}\left(X U^{t_{j}^{\prime}} X U^{-t_{j}^{\prime}} U^{r}\right)
\end{aligned}
$$




$$
\begin{aligned}
& =\sum_{j} \phi\left(b_{0}^{\prime} X_{t_{1}^{\prime}} \ldots X_{t_{j}^{\prime}} b_{j}^{\prime}\right) \phi\left(b_{j+1}^{\prime} X_{t_{j+2}^{\prime}} \ldots X_{t_{n}^{\prime}} b_{n}^{\prime}\right) \cdot \eta_{X}\left(U^{t_{j}^{\prime}}\right) U^{r-t_{j}^{\prime}} \\
& =\sum_{j} \eta_{X} \circ E^{\phi}\left(b_{0}^{\prime} X_{t_{1}^{\prime}} \ldots X_{t_{j}^{\prime}} b_{j}^{\prime} U^{t_{j}^{\prime}}\right) \cdot E^{\phi}\left(U^{r-t_{j}^{\prime}} b_{j+1}^{\prime} X_{t_{j+2}^{\prime}} \ldots X_{t_{n}^{\prime}} b_{n}^{\prime}\right) \\
& =\sum_{j}\left\langle 1 \otimes_{\eta} 1, b_{0}^{\prime} X_{t_{1}^{\prime}} \ldots X_{t_{j}^{\prime}} b_{j}^{\prime} U^{t_{j}^{\prime}} \otimes U^{r-t_{j}} b_{j+1}^{\prime} X_{t_{j+2}^{\prime}} \ldots X_{t_{n}^{\prime}} b_{n}^{\prime}\right\rangle_{\eta} \\
& =\sum_{j}\left\langle 1 \otimes_{\eta} 1, b_{0} U^{s_{1}} X_{t_{1}} \ldots X_{t_{j}} b_{j} U^{s_{j}} U^{t_{j}^{\prime}} \otimes\right. \\
& \left.\left.=\sum_{j}^{r-t_{j}} U^{s_{j+1}} b_{j+1} X_{t_{j+2}} \ldots X_{t_{n}} b_{n} U^{s_{n}} U^{-r}\right\rangle_{\eta} \otimes_{\eta} 1, b_{0} U^{s_{1}} X_{t_{1}} \ldots X_{t_{j}} b_{j} U^{s_{j}} \otimes U^{s_{j+1}} b_{j+1} X_{t_{j+2}} \ldots X_{t_{n}} b_{n} U^{s_{n}}\right\rangle_{\eta} \\
& =\left\langle 1 \otimes_{\eta} 1, \delta_{X}(Q)\right\rangle_{\eta} .
\end{aligned}
$$

Conversely, assume that $\zeta$ satisfying (3.1) exists. Since the argument above is reversible, it is sufficient to prove that $\zeta$ is in the image of $i$ : $L^{2}(M) \rightarrow L^{2}\left(P, E^{\phi}\right)$. Let $\theta_{t}$ be the dual action of $\mathbb{R}$ on $P$, given by $\theta_{t}\left(U_{s}\right)=$ $\exp (2 \pi i s t), \theta_{t}(m)=m, m \in M$. It is sufficient to prove that $\theta_{t}(\zeta)=\zeta$, since $i\left(L^{2}(M)\right)$ consists precisely of those vectors, which are left fixed by $\theta$. It is sufficient to prove that $\theta_{s}\left(E^{\phi}\left(\zeta m U_{t}\right)\right)=\exp (2 \pi i s t)$ if $m \in M$. Since $\zeta$ is assumed to be in the closure of $B[X] \vee L(\mathbb{R})$, it is sufficient to check this for $m \in B[X]$. But then by (3.1),

$$
\begin{aligned}
E^{\phi}\left(\zeta m U_{t}\right) & =\left\langle\zeta, m U_{t}\right\rangle_{L(\mathbb{R})} \\
& =\left\langle 1 \otimes_{\eta} 1, \delta_{X}(m U)\right\rangle_{\eta} \\
& =\left\langle 1 \otimes_{\eta} 1, \delta_{X}(m)\right\rangle_{\eta} U \\
& \in M \cdot U_{t},
\end{aligned}
$$

which gives the desired result, since $\theta_{s}$ acts trivially on $M$.

Note that (3.1) means that $\zeta$ is equal to $J(X: B \vee L(\mathbb{R}), \eta)$ in the notation of $[7]$. (This is strictly speaking incorrect, since the setting of $[7]$ presumes the existence of a finite trace on $B[X] \vee L(\mathbb{R})$; however, it is not hard to check that the arguments in [7] go through also in the case of a semifinite trace, which exists in our case.)

This fact has many consequences for the conjugate variables $J_{\phi}(X: B)$, coming from the properties of $J(X: B \vee L(\mathbb{R}), \eta)$. Note in particular that if $W^{*}\left(D,\left\{\sigma_{t}^{\phi}(X): t \in \mathbb{R}\right\}\right)$ is free from $B$ with amalgamation over $D \subset B$ with respect to some conditional expectation $E: B \rightarrow D$, and $E$ is $\phi$-preserving, then $X$ is free from $B \vee L(\mathbb{R})$ with amalgamation over $D \vee L(\mathbb{R})$ (see [12], $[6])$. We record this as: 
Theorem 3.2. Assume that $E: B \rightarrow D$ is a $\phi$-preserving conditional expectation. If $W^{*}\left(D,\left\{\sigma_{t}^{\phi}(X): t \in \mathbb{R}\right\}\right)$ is free from $B$ over $D$, then

$$
J_{\phi}(X: B)=J_{\phi}(X: D) .
$$

In a similar way, one can generalize to $J_{\phi}(X: B)$ all the properties of the conjugate variable $J(X: B, \eta)$ proved in [7].

Reformulating gives the following properties of $\Phi_{\phi}$, which we list for reader's convenience, since they are needed in the rest of the paper:

Theorem 3.3. Let $\phi$ be a normal faithful state on $M, B \subset M$ be globally fixed by the modular group (i.e., $\sigma_{t}^{\phi}(B)=B$ for all $t$ ), and $X_{i} \in M$. Then:

(a) $\Phi_{\phi}^{*}\left(\lambda X_{1}, \ldots, \lambda X_{n}: B\right)=\lambda^{-2} \Phi_{\phi}^{*}\left(X_{1}, \ldots, X_{n}: B\right)$ for all $\lambda \in \mathbb{R} \backslash\{0\}$.

(b) If $B \subset A \subset M$ and $A$ is globally fixed by $\sigma^{\phi}$, then $\Phi_{\phi}^{*}\left(X_{1}, \ldots, X_{n}\right.$ : $A) \geq \Phi^{*}\left(X_{1}, \ldots, X_{n}: B\right)$.

(c) If $C \subset M$ is globally fixed by $\sigma^{\phi}$, and $W^{*}\left(X_{1}, \ldots, X_{n}\right)$ and $B$ are free with amalgamation over $C$ (with respect to the unique $\phi$-preserving conditional expectation from $M$ onto $C)$, then $\Phi_{\phi}^{*}\left(X_{1}, \ldots, X_{n}: B \vee\right.$ $C)=\Phi_{\phi}^{*}\left(X_{1}, \ldots, X_{n}: C\right)$.

(d) If $Y_{i} \in M$ are self-adjoint, $D \subset B, D \subset C$ subalgebras of $M$, which are globally fixed by $\sigma^{\phi}$, and $B\left[X_{1}, \ldots, X_{n}\right]$ is free from $C\left[X_{1}, \ldots, X_{n}\right]$ over $D$ (with respect to the unique $\phi$-preserving conditional expectation from $M$ onto $D)$, then $\Phi_{\phi}^{*}\left(X_{1}, \ldots, X_{n}, Y_{1}, \ldots, Y_{m}: B \vee C\right)=$ $\Phi_{\phi}^{*}\left(X_{1}, \ldots, X_{n}: B\right)+\Phi_{\phi}^{*}\left(Y_{1}, \ldots, Y_{n}: C\right)$.

(e) $\Phi_{\phi}^{*}\left(X_{1}, \ldots, X_{n}, Y_{1}, \ldots, Y_{n}: B\right) \geq \Phi_{\phi}^{*}\left(X_{1}, \ldots, X_{n}: B\right)+\Phi_{\phi}^{*}\left(Y_{1}, \ldots\right.$, $\left.Y_{m}: B\right)$.

(f) $\Phi_{\phi}^{*}\left(X_{1}, \ldots, X_{n}: B\right) \cdot \phi\left(\sum X_{i}^{*} X_{i}\right)^{2} \geq n^{2}$. Equality holds iff $\left\{\sigma_{t_{1}}^{\phi}\left(X_{1}\right)\right.$, $\left.\ldots, \sigma_{t_{n}}^{\phi}\left(X_{n}\right): t_{1}, \ldots, t_{n} \in \mathbb{R}\right\}$ have the same distribution as the semicircular family $\left\{\kappa s\left(\sigma_{t_{1}}^{\phi}\left(X_{1}\right)\right), \ldots, \kappa s\left(\sigma_{t_{n}}^{\phi}\left(X_{n}\right)\right): t_{1}, \ldots, t_{n} \in \mathbb{R}\right\}$ with respect to the free quasi-free state, $\kappa>0$.

We mention that all of the statements in Sections 3 and 4 of [7] remain valid for $\Phi_{\phi}^{*}$; we leave details to the reader.

One can also define and study free entropy $\chi_{\phi}^{*}\left(X_{1}, \ldots, X_{n}\right)$ by setting $X_{i}^{\varepsilon}=X_{i}+\sqrt{\varepsilon} Y_{i}$ to be the free Brownian motion described in the beginning of the paper, and letting

$$
\chi_{\phi}^{*}\left(X_{1}, \ldots, X_{n}\right)=\frac{1}{2} \int_{0}^{\infty}\left(\frac{n}{1+t}-\Phi_{\phi}^{*}\left(X_{1}^{t}, \ldots, X_{n}^{t}\right)\right) d t .
$$

The properties of $\chi^{*}(\ldots, \eta)$ once again generalize to $\chi_{\phi}^{*}$ (compare Section 8 of $[7])$. 


\section{States on a $\mathrm{II}_{1}$ factor.}

4.1. $\Phi_{\phi}^{*}$ vs. $\Phi_{\tau}^{*}$. The following theorem is somewhat surprising, since it shows that $\Phi_{\phi}^{*}$ is identically infinite for most states $\phi$ on a $\mathrm{II}_{1}$ factor (the analogy with classical Fisher information would instead suggest that $\phi \mapsto \Phi_{\phi}^{*}$ would have some nice convexity properties). This, on the other hand, goes well with the "degenerate convexity" property of the microstates free entropy $\chi[\mathbf{1 5}]$ (which is reflected in that it is identically $-\infty$ on generators of any von Neumann algebra with more than one unital trace).

Theorem 4.1. Let $M$ be a tracial von Neumann algebra, $\phi$ a faithful normal state on $M, B \subset M$ a subalgebra so that $\sigma_{t}^{\phi}(B)=B$ for all $t$, and $X=X^{*} \in M$. Then if $J_{\phi}(X: B)$ exists, the modular group of $\phi$ must fix $X$.

Proof. Let $d \in M$ be a positive element, so that $\phi(x)=\tau(d x)$, where $\tau$ is a normal faithful trace on $M$, and $d$ is an unbounded operator on $L^{2}(M, \tau)$, affiliated to $M$. The modular group of $\phi$ is then given by $\sigma_{t}^{\phi}(x)=d^{i t} x d^{-i t}$, $x \in M$. Denoting by $X_{t}$ the element $\sigma_{t}^{\phi}(X)$, we then get

$$
X=X_{0}=d^{-i t} X_{t} d^{i t}, \quad t \in \mathbb{R} .
$$

Consider

$$
\phi\left(X_{0}^{2}\right)=\phi\left(J_{\phi}(X: B) \cdot X_{0}\right)=\phi\left(J_{\phi}(X: B) d^{-i t} X_{t} d^{i t}\right) .
$$

Let $b_{1}$ and $b_{2}$ be two elements in the domain of $\partial_{X}$, so that $b_{1}=b_{2}^{*}$. Then we get, writing $Y_{t}=\sigma_{t}^{\hat{\phi}}(Y)$ :

$$
\begin{aligned}
\phi\left(J_{\phi}(X: B) b_{1} X_{t} b_{2}\right)= & \hat{\phi}\left(Y_{0} b_{1} Y_{t} b_{2}\right) \\
& +\hat{\phi}\left(Y_{0} \partial_{X}\left(b_{1}\right) X_{t} b_{2}\right)+\hat{\phi}\left(Y_{0} b_{1} X_{t} \partial_{X}\left(b_{2}\right)\right) \\
= & \phi\left(b_{1}\right) \phi\left(b_{2}\right) \hat{\phi}\left(Y_{0} Y_{t}\right) \\
& +\hat{\phi}\left(Y_{0} \partial_{X}\left(b_{1}\right) X_{t} b_{2}\right)+\hat{\phi}\left(\left[\partial_{X}\left(b_{2}^{*}\right) X_{t} b_{1}^{*} Y_{0}\right]^{*}\right) \\
= & \phi\left(b_{1}\right) \phi\left(b_{1}^{*}\right) \hat{\phi}\left(Y_{0} Y_{t}\right) \\
& +\hat{\phi}\left(Y_{0} \partial_{X}\left(b_{1}\right) X_{t} b_{1}^{*}\right)+\hat{\phi}\left(\left[\partial_{X}\left(b_{1}\right) X_{t} b_{1}^{*} Y_{0}\right]^{*}\right) .
\end{aligned}
$$

Now, for all $m, n \in M$, we have

$$
\hat{\phi}\left(Y_{0} m Y_{0} n\right)=\phi(m) \phi(n)=\hat{\phi}\left(m Y_{0} n Y_{0}\right),
$$


so that

$$
\begin{aligned}
& \hat{\phi}\left(Y_{0} \partial_{X}\left(b_{1}\right) X_{t} b_{1}^{*}\right)+\hat{\phi}\left(\left[\partial_{X}\left(b_{1}\right) X_{t} b_{1}^{*} Y_{0}\right]^{*}\right) \\
& =\hat{\phi}\left(Y_{0} \partial_{X}\left(b_{1}\right) X_{t} b_{1}^{*}\right)+\hat{\phi}\left(\left[Y_{0} \partial_{X}\left(b_{1}\right) X_{t} b_{1}^{*}\right]^{*}\right) \\
& =\hat{\phi}\left(Y_{0} \partial_{X}\left(b_{1}\right) X_{t} b_{1}^{*}\right)+\overline{\hat{\phi}\left(Y_{0}\left[\partial_{X}\left(b_{1}\right) X_{t} b_{1}^{*}\right]\right)} \\
& \in \mathbb{R} .
\end{aligned}
$$

It follows that

$$
\Im \phi\left(J_{\phi}(X: B) b_{1} X_{t} b_{1}^{*}\right)=\Im \hat{\phi}\left(Y_{0} b_{1} Y_{t} b_{1}^{*}\right) .
$$

Now fix $t \in \mathbb{R}$ and choose $a_{n}$ in the domain of $\partial_{X},\left\|a_{n}\right\| \leq 1$, so that

$$
a_{n} \rightarrow d^{i t}, \quad a_{n}^{*} \rightarrow d^{-i t} \quad \text { strongly. }
$$

One can choose $a_{n}$, for example, to be elements of the algebra $B[X]$. Then

$$
\begin{aligned}
0=\Im \phi\left(X_{0}^{2}\right) & =\Im \phi\left(J_{\phi}(X: B) \cdot X_{0}\right) \\
& =\Im \phi\left(J_{\phi}(X: B) d^{-i t} X_{t} d^{i t}\right) \\
& =\lim _{n \rightarrow \infty} \Im \phi\left(J_{\phi}(X: B) a_{n} X_{t} a_{n}^{*}\right) \\
& =\lim _{n \rightarrow \infty} \Im \hat{\phi}\left(Y_{0} a_{n} Y_{t} a_{n}^{*}\right) \\
& =\lim _{n \rightarrow \infty} \Im\left(\phi\left(a_{n}\right) \phi\left(a_{n}^{*}\right) \hat{\phi}\left(Y_{0} Y_{t}\right)\right) \\
& =\lim _{n \rightarrow \infty} \phi\left(a_{n}\right) \phi\left(a_{n}^{*}\right) \Im \hat{\phi}\left(Y_{0} Y_{t}\right) \\
& =\phi\left(d^{i t}\right) \phi\left(d^{-i t}\right) \Im \hat{\phi}\left(Y_{0} Y_{t}\right) .
\end{aligned}
$$

Since $\hat{\phi}\left(Y_{0} Y_{t}\right)=\phi\left(X_{0} X_{t}\right)$, for $t$ sufficiently close to zero (so that $\phi\left(d^{i t}\right) \neq 0$ ), we get that

$$
\phi\left(X X_{t}\right) \in \mathbb{R}
$$

Thus

$$
\begin{aligned}
0 & =\tau\left(d X X_{t}\right)-\tau\left(d\left(X X_{t}\right)^{*}\right) \\
& =\tau\left(d X d^{i t} X d^{-i t}-d^{i t} X d^{-i t} X d\right) \\
& =\tau\left((d X-X d) d^{i t} X d^{-i t}\right) \\
& =\tau\left([d, X] d^{i t} X d^{-i t}\right) .
\end{aligned}
$$

Differentiating this in $t$, and noting that $(d / d t)_{t=0}\left(d^{i t} X d^{-i t}\right)=i[d, X]$ gives

$$
i \tau\left([d, X]^{2}\right)=0 \text {. }
$$

Since $[d, X]$ is anti-self-adjoint, this implies that $\tau\left(|[d, X]|^{2}\right)=0$, so that $[d, X]=0$, because $\tau$ is faithful. This means that $\sigma_{t}^{\phi}(X)=X$ for all $t$. 
Corollary 4.2. Suppose that $X_{1}, \ldots, X_{n}$ are self-adjoint generators of a $\mathrm{II}_{1}$ factor $M$. Let $\phi$ be a normal faithful state on $M$, and denote by $\tau$ the unique faithful normal trace on $M$. Then $\Phi_{\phi}^{*}\left(X_{1}, \ldots, X_{n}\right)<+\infty$ implies that:

(1) $\Phi_{\tau}^{*}\left(X_{1}, \ldots, X_{n}\right)<\infty$, and

(2) $\phi$ is a multiple of the trace $\tau$ on $M$.

Proof. Clearly, the second statement implies the first. To get the second statement, write $\phi(\cdot)=\tau(d \cdot)$ and apply the theorem to conclude that $\left[d, X_{i}\right]=0$. Since $X_{1}, \ldots, X_{n}$ generate $M, d$ must be in the center of $M$, which must consist of multiples of identity, since $M$ is a factor. But then $d$ is a scalar multiple of identity, so that $\phi$ and $\tau$ are proportional.

4.2. Factoriality. Voiculescu showed [15] that for his microstates entropy $\chi$ the following implication holds:

$$
\chi\left(X_{1}, \ldots, X_{n}\right)>-\infty \Rightarrow W^{*}\left(X_{1}, \ldots, X_{n}\right) \text { is a factor. }
$$

In fact, the conclusion is stronger: Not only is the center of $W^{*}\left(X_{1}, \ldots, X_{n}\right)$ trivial, but so is its asymptotic center. Unfortunately, we don't know if the same implication holds for the non-microstates free entropy $\chi^{*}$ introduced by Voiculescu in [16], or even under the stronger assumption that $\Phi^{*}\left(X_{1}, \ldots, X_{n}\right)$ is finite. We prove a weaker version of the assertion above for $\Phi^{*}=\Phi_{\tau}^{*}$. We first need a technical lemma:

Lemma 4.3. Let $\phi$ be a normal faithful state on $M$. Let $X \in M$ be selfadjoint and $B \subset M$ be a subalgebra, so that $\sigma_{t}^{\phi}(B)=B$ for all $t$. Assume that $p \in B$ is a self-adjoint projection, $\phi(p)=\alpha$, and so that $\sigma_{t}^{\phi}(p)=p$ for all t. Assume that $\|[X, p]\|_{2}<\delta$. Then

$$
\Phi_{\phi}^{*}(X: B)>4 \frac{\alpha^{2}(1-\alpha)^{2}}{\delta^{2}} .
$$

Proof. Let $(A, \tau)$ be a copy of $L\left(\mathbb{F}_{2}\right)$, free from $B[X]$. Since $\Phi_{\phi}^{*}(X: B)=$ $\Phi_{\phi}^{*}(X: B \vee A)$, and since the centralizer of $\left\{\sigma_{t}^{\phi}(B)\right\}_{t} \vee A$ is a factor [1], we can find a projection $q \in B \vee A$, which is fixed by the modular group, and so that $\|[X, q]\|_{2}<\delta$, and $\tau(q)=\beta=m / n$ is rational and close to $\alpha$. We may moreover find a family of matrix units $e_{i j} \in B \vee A, 1 \leq i, j \leq n$, fixed by the modular group, and so that

$$
\begin{aligned}
e_{i j}^{*} & =e_{j i}, \quad e_{i j} e_{k l}=\delta_{j k} e_{i l} \\
\tau\left(e_{i i}\right) & =\frac{1}{n}, \quad q=\sum_{i=1}^{m} e_{i i} .
\end{aligned}
$$

Denote by $C$ the algebra generated in $B \vee A$ by $\left\{e_{i j}\right\}_{1 \leq i, j \leq n}$. Note that $C \cong M_{n \times n}$, the algebra of $n \times n$ matrices. The restriction of $\phi * \tau$ to $C$ is the usual matrix trace. Then

$$
\Phi_{\phi}^{*}(X: B)=\Phi_{\phi * \tau}^{*}(X: B \vee A) \geq \Phi_{\phi * \tau}^{*}(X: C) .
$$


Write $X_{i j}=e_{1 i} X e_{j 1}$. Then the inequality $\|[X, q]\|_{2}<\delta$ implies that

$$
\begin{aligned}
\delta & >\|x q-q x\|_{2} \\
& =\|q x q+(1-q) x q-q x q-q x(1-q)\|_{2} \\
& =\|(1-q) x q-q x(1-q)\|_{2} \\
& =\sqrt{2} \cdot\|q x(1-q)\|_{2},
\end{aligned}
$$

since $(1-q) x q$ and $q x(1-q)$ are orthogonal. Hence

$$
\|q X(1-q)\|_{2}<\delta / \sqrt{2}
$$

It follows that

$$
\sum_{1 \leq i \leq m, m<j \leq n} \phi\left(X_{i j}^{*} X_{i j}\right)+\sum_{m<i \leq n, 1 \leq i \leq n} \phi\left(X_{i j}^{*} X_{i j}\right)<\delta^{2} .
$$

Denote by $\phi^{\prime}$ the state $n(\phi * \tau)\left(e_{11} \cdot e_{11}\right)$ on $e_{11} W^{*}(X, C) e_{11}$. Then

$$
\begin{aligned}
\Phi_{\phi^{\prime}}^{*}\left(\left\{X_{i j}\right\}\right) & \geq \sum_{i, j} \Phi_{\phi^{\prime}}^{*}\left(\left\{X_{i j}\right\}\right) \\
& >2 m(n-m) \frac{1}{n\left(\delta^{2} / 2 m(n-m)\right)} \\
& =\frac{(2 m(n-m))^{2}}{n \delta^{2}} \\
& =n^{3} 4 \frac{\beta^{2}(1-\beta)^{2}}{\delta^{2}} .
\end{aligned}
$$

Arguing exactly as in [4, Proposition 4.1], we get that

$$
\Phi_{\phi * \tau}^{*}(X: C)=\frac{1}{n^{3}} \Phi_{\phi^{\prime}}^{*}\left(\left\{X_{i j}\right\}\right)>4 \frac{\beta^{2}(1-\beta)^{2}}{\delta^{2}} .
$$

Since $\beta$ was a rational number, arbitrarily close to $\alpha$, we get the desired estimate for $\Phi_{\phi}^{*}(X: B)$.

Theorem 4.4. Assume that $M$ is a von Neumann algebra with a faithful normal trace $\tau$, and $X_{i}$ are a family of self-adjoint elements in $M,\left\|X_{i}\right\|=1$. Assume that $B_{i}$ form an increasing sequence of subalgebras of $M$, so that $M={\overline{\cup B_{i}}}^{w}$. Assume further that for some normal faithful state $\phi$ on $M$,

$$
\sup _{j} \liminf _{i} \Phi_{\phi}^{*}\left(X_{i}: B_{j}\right)<+\infty .
$$

Then $M$ is a factor.

Proof. In view of Theorem 4.1, we may assume that $\phi$ is a trace, $\tau$. Assume that $M$ is not a factor. Then there exists a central projection $p \in M$ of some trace $\alpha=\tau(p), \alpha(1-\alpha) \neq 0$. Moreover, $\left[p, X_{i}\right]=0$ for all $i$. Since $B_{i}$ 
increase to all of $M$, given $\delta>0$, there is a large enough $j$ and a projection $q \in B_{j}$, so that $\|q-p\|_{2}<\delta / 2$. Then for any $k$,

$$
\begin{aligned}
\left\|\left[q, X_{k}\right]\right\|_{2} & =\left\|q X_{k}-X_{k} q\right\|_{2} \\
& =\left\|(q-p) X_{k}-X_{k}(q-p)+p X_{k}-X_{k} p\right\|_{2} \\
& \leq\left\|(q-p) X_{k}\right\|_{2}+\left\|X_{k}(q-p)\right\|_{2}+0 \\
& \leq 2\|(q-p)\|_{2}\left\|X_{k}\right\| \\
& <2(\delta / 2)=\delta .
\end{aligned}
$$

Now applying Lemma 4.3, we deduce that for any $i>j$,

$$
\Phi_{\tau}^{*}\left(X_{i}: B_{j}\right)>4 \frac{\alpha^{2}(1-\alpha)^{2}}{\delta^{2}} .
$$

Hence $\liminf { }_{i} \Phi_{\tau}^{*}\left(X_{i}: B_{j}\right)>4 \alpha^{2}(1-\alpha)^{2} / \delta^{2}$, which is a contradiction, since $\delta$ was arbitrary.

The hypothesis of the theorem is satisfied for some von Neumann algebras. For example, let $M=L\left(\mathbb{F}_{\infty}\right)$ generated by an infinite semicircular family $X_{i}, i=1,2,3, \ldots$ Then if $B_{i}=W^{*}\left(X_{j}: j<i\right)$, the assumptions of the theorem are satisfied. In fact if $Y_{i}$ is any family of elements of a tracial von Neumann algebra, so that $\left\|Y_{i}\right\|=1$, and $X_{i}$ are a free semicircular family, then letting $Z_{i}(\varepsilon)=Y_{i}+\varepsilon X_{i}, M_{\varepsilon}=W^{*}\left(Z_{1}(\varepsilon), Z_{2}(\varepsilon), \ldots\right)$ and $B_{j}=$ $W^{*}\left(Z_{1}(\varepsilon), \ldots, Z_{j}(\varepsilon)\right)$, we see that $M_{\varepsilon}$ is a factor. In other words, generators of an arbitrary tracial von Neumann algebra can be perturbed (in a certain representation of this algebra) by an arbitrarily small amount $\varepsilon$ in uniform norm, to produce a $\mathrm{II}_{1}$ factor. Another way of putting it is to note that the free Brownian motion $\varepsilon \mapsto Z_{j}(\varepsilon)$ started at $\left\{Y_{1}, Y_{2}, \ldots\right\}$ generates a factor at any time $\varepsilon>0$.

4.3. Factoriality in the non-tracial case. In a similar way, we get the following:

Theorem 4.5. Assume that $M$ is a von Neumann algebra with a faithful normal state $\phi$, and $X_{i}$ are a family of self-adjoint elements in $M,\left\|X_{i}\right\|=1$. Assume that $B_{i}$ form an increasing sequence of subalgebras of $M, \sigma_{t}^{\phi}\left(B_{i}\right)=$ $B_{i}$ for all $t$ and $i$, and assume that $M^{\phi}={\overline{\cup\left(B_{i} \cap M^{\phi}\right)}}^{w}$. Let $R_{i}$ be the operator of right multiplication by $X_{i}$ densely defined on $L^{2}(M, \phi)$. Assume that $\sup _{i}\left\|R_{i}\right\|=C<+\infty$. Assume further that

$$
\sup _{j} \liminf _{i} \Phi_{\phi}^{*}\left(X_{i}: B_{j}\right)<+\infty .
$$

Then $M$ is a factor.

Proof. Assume that $M$ is not a factor. Then there exists a central projection $p \in M, \alpha=\phi(p), \alpha(1-\alpha) \neq 0$. Moreover, $\left[p, X_{i}\right]=0$ for all $i$. Since automatically $p \in M^{\phi}$ and $B_{i} \cap M^{\phi}$ increase to all of $M^{\phi}$, given $\delta>0$, there 
is a large enough $j$ and a projection $q \in B_{j} \cap M^{\phi}$, so that $\|q-p\|_{2}<\delta /(1+C)$. Then for any $k$,

$$
\begin{aligned}
\left\|\left[q, X_{k}\right]\right\|_{2} & =\left\|q X_{k}-X_{k} q\right\|_{2} \\
& =\left\|(q-p) X_{k}-X_{k}(q-p)+p X_{k}-X_{k} p\right\|_{2} \\
& \leq\left\|(q-p) X_{k}\right\|_{2}+\left\|X_{k}(q-p)\right\|_{2}+0 \\
& \leq\left\|R_{k}\right\|\|(q-p)\|_{2}+\|(q-p)\|_{2}\left\|X_{k}\right\| \\
& <(1+C)(\delta /(1+C))=\delta .
\end{aligned}
$$

The rest of the argument proceeds just like in the tracial case.

Note that the assumption on the norms of $R_{i}$ is satisfied if each $X_{i}$ is

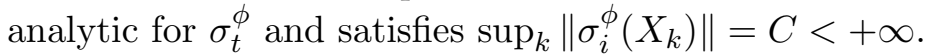

Acknowledgement. This work was supported by an NSF postdoctoral fellowship and NSF grant DMS-0102332. The author is grateful to MSRI and the organizers of the operator algebras program for their hospitality.

\section{References}

[1] L. Barnett, Free products of von Neumann algebras of type III, Proc. Amer. Math. Soc., 123 (1995), 543-553, MR 95c:46096, Zbl 0808.46088.

[2] L. Ge, Applications of free entropy to finite von Neumann algebras II, Ann. of Math. (2), 147(1) (1998), 143-157, MR 99c:46068, Zbl 0924.46050.

[3] A. Guionnet, Large deviations upper bounds and central limit theorems for noncommutative functionals of Gaussian large random matrices, Ann. Inst. H. Poincaré Probab. Statist., 38 (2002), 341-384, MR 2003a:60042, Zbl 0995.60028.

[4] A. Nica, D. Shlyakhtenko and R. Speicher, Some minimization problems for the free analogue of the Fisher information, Adv. Math., 121 (1999), 282-321, MR 2000b:46120, Zbl 0929.46052.

[5] D. Shlyakhtenko, Free quasi-free states, Pacific J. Math., 177 (1997), 329-368, MR 98b:46086, Zbl 0882.46026.

[6] _ A-valued semicircular systems, J. Func. Anal., 166 (1999), 1-47, MR 2000j:46124, Zbl 0951.46035.

[7] _ Free entropy with respect to a completely positive map, Amer. J. Math., 122(1) (2000), 45-81, MR 2001f:46102, Zbl 0949.46033.

[8] _ A microstates approach to relative free entropy, Internat. J. Math., 13 (2002), 605-623, MR 2003h:46096.

[9] , Prime type III factors, Proc. Natl. Acad. Sci. USA, 97 (2000), 12439-12441, MR 2002c:46121, Zbl 0969.46043.

[10] M. Stefan, Idecomposibility of free group factors over nonprime subfactors and abelian subalgebras. Preprint, 1999.

[11] Ş. Strătilă, Modular Theory in Operator Algebras, Editura Academiei, Bucharest and Abacus Press, England, 1981, MR 85g:46072, Zbl 0504.46043. 
[12] Y. Ueda, A minimal action of the compact quantum group $\mathrm{SU}_{q}(n)$ on a full factor, J. Math. Soc. Japan, 51(2) (1999), 449-461, MR 2000c:46127, Zbl 0928.46049.

[13] D.-V. Voiculescu, The analogues of entropy and of Fisher's information measure in free probability theory I, Commun. Math. Phys., 155 (1993), 71-92, MR 94k:46137, Zbl 0781.60006.

[14] - The analogues of entropy and of Fisher's information measure in free probability theory, II, Invent. Math., 118 (1994), 411-440, MR 96a:46117, Zbl 0820.60001.

[15] _ The analogues of entropy and of Fisher's information measure in free probability theory, III, Geom. Funct. Anal., 6 (1996), 172-199, MR 96m:46119, Zbl 0856.60012.

[16] _ The analogues of entropy and of Fisher's information measure in free probabilility, V, Invent. Math., 132 (1998), 189-227, MR 99d:46087, Zbl 0930.46053.

[17] D.-V. Voiculescu, K. Dykema and A. Nica, Free Random Variables, CRM Monograph Series, 1, Amer. Math. Soc., Providence, 1992, MR 94c:46133, Zbl 0795.46049.

Received March 5, 2002 and revised July 22, 2002.

Department of Mathematics

University of California, Los Angeles

Los Angeles, CA 90095

E-mail address: shlyakht@math.ucla.edu 